\title{
THE a-SiC/c-Si(n) ISOTYPE HETEROJUNCTION AS A HIGH SENSITIVITY TEMPERATURE SENSOR
}

\author{
L. MAGAFAS*, N. GEORGOULAS and A. THANAILAKIS ${ }^{1}$ \\ Laboratory of Electrical and Electronic Material Technology, \\ Department of Electrical and Computer Engineering, Democritous \\ University of Thrace, 67100, Xanthi, Greece
}

(Received 10 September 1997; In final form 3 November 1997)

\begin{abstract}
The a-SiC/c-Si(n) isotype heterojunction has been studied as a temperature sensor by measuring its reverse current-voltage $\left(I_{R^{-}} V\right)$ and reverse voltage-temperature $(V-T)$ characteristics, as well as its reverse current temperature dependence. The $I_{R^{-}} V$ characteristics exhibit an almost constant current, whereas the reverse current $I_{R}$ depends strongly on $T$ (from $230 \mathrm{~K}$ up to $320 \mathrm{~K}$ ). The $V-T$ characteristics, at different reverse currents, reveal a highly temperature sensitive behavior for the a-SiC/c-Si $(n)$ junction. The measured values of temperature sensitivity $(\Delta V / \Delta T)_{\max }$ was found to be $(\cong-2.5 \mathrm{~V} / \mathrm{K})$ in the moderate temperature range, which are much higher than those obtained with bulk semiconductor temperature sensors. The high sensitivity-temperature-range of the a-SiC/c-Si $(n)$ heterojunctions can be controlled electrically within the regim of values from $230 \mathrm{~K}$ up to $320 \mathrm{~K}$. Finally, the high sensitivity of these devices, in conjunction with the fact that a-SiC films can be used as an add-on to the existing $\mathrm{Si}$ technology, emerge new possibilities to the fabrication of high sensitivity temperature microsensors.
\end{abstract}

Keywords: Heterojunction; temperature sensors; microelectronic devices

\section{INTRODUCTION}

The interest in developing high sensitivity semiconductor temperature sensors has been increased during the past few years [1]. If integrated with semiconductor microelectronic circuitry, such sensors could form the basis for a new generation of sensing devices. Although many

${ }^{*}$ Corresponding author. 
crystalline microelectronic devices have received considerable attention as temperature sensors, e.g. $p-n$ diodes [2], transistors [3], Bulk Barrier Diodes (BBD) [1] etc., none of them, except from BBD, was found to exhibit a high sensitivity in the range of moderate temperatures.

On the other hand, although a number of other microelectronic devices have been fabricated using a-Si:H or a-SiC:H, e.g. MetalAmorphous Silicon FETs [4, 5], thin film Transistors [6], Optothyristors [7], and Heterojunction Phototransistors [8] there is no published work in the area of temperature sensors using amorphous semiconductors. Taking, also, into account that localized gap states have a crucial effect on the electrical properties of amorphous semiconductor materials $[9,10]$ and devices [11], it seems to be a great need to study temperature sensor devices based on amorphous semiconductors.

A contribution in this particular direction is the present work, presenting results on the behaviour of a-SiC/c-Si $(n)$ isotype heterojunctions as highly sensitive temperature sensors, obtained by measuring their reverse current-voltage characteristics at different temperatures, as well as their reverse voltage-temperature characteristics using a current source. The results of these measurements reveal that the a-SiC/c-Si $(n)$ isotype heterojunction is a very attractive device as a high sensitivity temperature sensor, with a sensitivity temperaturerange electrically controlled within the region of values from $230 \mathrm{~K}$ up to $320 \mathrm{~K}$.

\section{FABRICATION OF a-SiC/c-Si(n) HETEROJUNCTIONS}

Thin films of a-SiC, with a thickness of about $1 \mu \mathrm{m}$, were deposited by r.f sputtering on $n$-type crystalline silicon substrates $(5-10 \Omega \mathrm{cm})$, with ohmic contacts at their back sides. The target used was SiC of constant composition ( $66 \mathrm{wt} \% \mathrm{Si} 34 \mathrm{wt} \% \mathrm{C}$ ) and $99.8 \%$ purity. The RF power was $250 \mathrm{~W}$ and the target-to-substrate distance was $5.5 \mathrm{~cm}$. The substrate temperature was $30^{\circ} \mathrm{C}$ and the sputtering chamber evacuated to a pressure lower than $5 \times 10^{-7}$ Torr before the introduction of argon. During the deposition, the flow rate of argon was $20 \mathrm{sccm}$ and the pressure in the sputtering chamber $6 \mathrm{~m}$ Torr. Aluminium dots, $5000^{\circ} \mathrm{A}$ 
thick and $0.785 \mathrm{~mm}^{2}$ in area, were deposited on the a-SiC layer to form ohmic contacts, as it has been described in our previous works $[12,13]$

The reverse current-voltage $\left(I_{R^{-}} V\right)$ and voltage-temperature measurements $(V-T)$ of a-SiC/c-Si $(n)$ isotype heterojunctions were carried out within a light-tight cryostat evacuated down to $10^{-3}$ Torr, in the temperature range from $230 \mathrm{~K}$ up to $320 \mathrm{~K}$, using a picoameter and a current source controlled by a P.C.

\section{RESULTS AND DISCUSSION}

Figure 1 presents a typical structure of a-SiC/c-Si $(n)$ heterojunctions (Fig. 1(a)) and the energy band diagram under thermal equilibrium conditions, as well as under reverse bias conditions (Fig. 1(b)). The above energy band diagram was calculated, in our previous study [13], using Anderson's model [14]. The conductivity of a-SiC was considered to be $n$-type, as it was found in our previous works [12, 13]. The subscripts 1 and 2 refer to a-SiC and to c-Si(n) respectively, so that $E_{\mathrm{c} 1}$ and $E_{\mathrm{c} 2}$ are the edges of the conduction bands, $E_{\mathrm{v} 1}, E_{\mathrm{v} 2}$ the edges of the valence bands, $\delta_{1}, \delta_{2}$ the distances between the Fermi level and the corresponding conduction band edges, $E_{\mathrm{g} 1}, E_{\mathrm{g} 2}$ the energy band gaps, $V_{b i 1}, V_{b i 2}$ the partial diffusion potentials, $\Delta E_{\mathrm{c}}, \Delta E_{\mathrm{v}}$, the discontinuities of the conduction and valence bands, respectively, and $E_{\mathrm{F}}$ the Fermi level under thermal equilibrium conditions. Under reverse bias conditions, the polarity is negative on the a-SiC side of the junction. As it is shown in Figure 1(b), under reverse bias conditions electrons from a-SiC have to surpass the potential barrier of the a-SiC/ c-Si $(n)$ junction in order to contribute to the reverse current of the heterojunction.

Figure 2 shows typical reverse $\log \left(I_{R}\right)-V$ characteristics of a-SiC/c$\mathrm{Si}(n)$ heterojunctions, measured at different temperatures from $230 \mathrm{~K}$ up to $320 \mathrm{~K}$. As it clear from this figure, the reverse current, due to the electron transport from a-SiC to c-Si $(n)$, increases with the increase of temperature. On the other hand, for a given temperature, $\log \left(I_{R}\right)$ remains almost constant with the increase of reverse bias voltage, exhibiting a saturation behavior. This behavior of the reverse current is very interesting (for temperature sensing devices), as it will be discussed further below, and it could be attributed to the charge carrier 


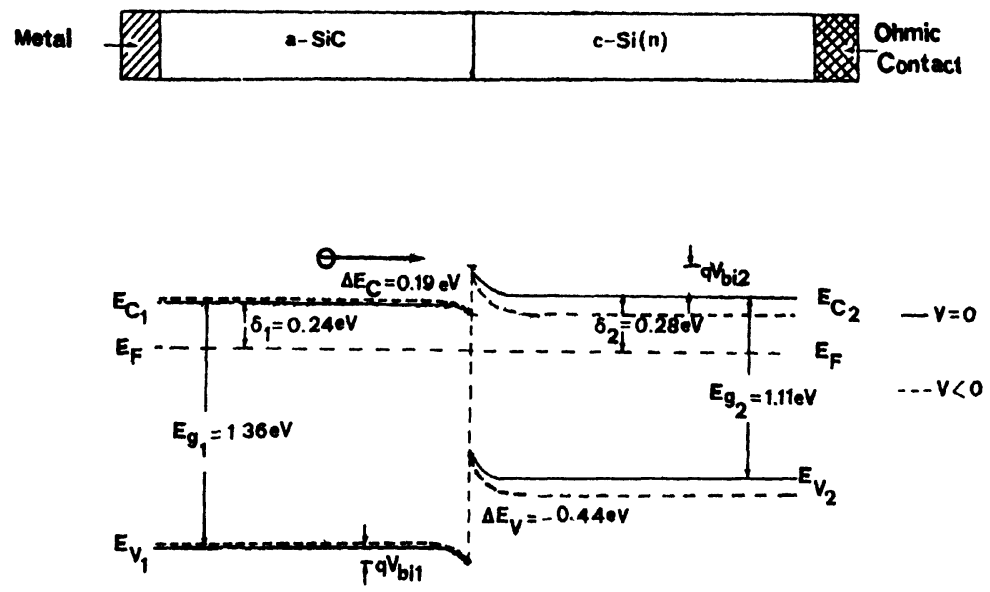

FIGURE 1 (a) Typical structure of a-SiC/c-Si(n) heterojunctions (b) Energy band diagram of a-SiC/c-Si $(n)$ heterojunctions under thermal equilibrium, $V=0(-)$, and reverse bias, $V<0(---)$, conditions.

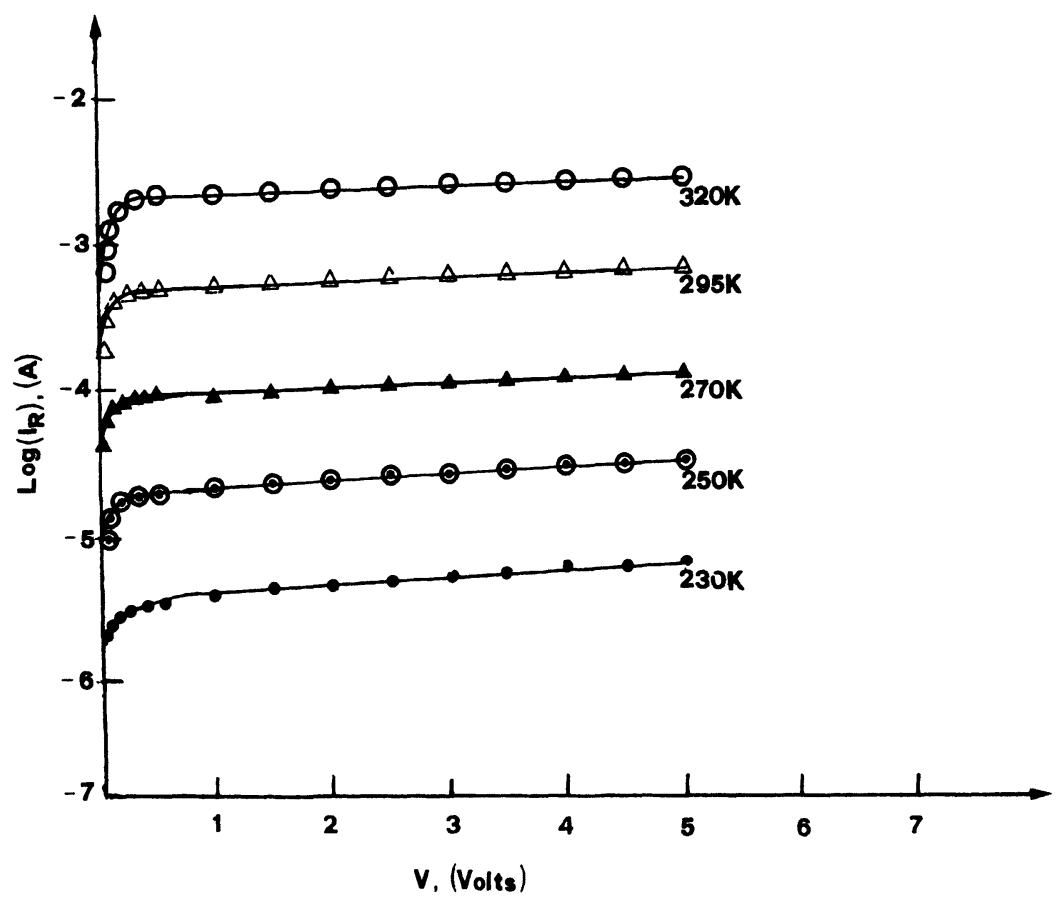

FIGURE 2 Typical reverse current-voltage $\left(\log \left(I_{R}\right)-V\right)$ characteristics of a-SiC/c-Si $(n)$ heterojunctions at different temperatures (device area $=0.785 \mathrm{~mm}^{2}$ ). 
transport mechanism, in combination with the fact that one side of the heterojunction consists of an amorphous semiconductor (a-SiC).

In an amorphous semiconductor like a-SiC (Fig. 1(b)), it is well known that when $\mathrm{E}_{\mathrm{F}}$ lies below $E_{\mathrm{c}}$ and the temperature is roughly higher than $230 \mathrm{~K}$ electrons in amorphous semiconductor are transported through extended states of the conduction band $[12,15]$. Thus, as we can see from Figure 1(b) under reverse bias conditions, electrons in extended states of the conduction band of a-SiC may be emitted over the barrier into the conduction band of $\mathrm{c}-\mathrm{Si}(n)$. In this case, for which thermionic emission is the dominant transport mechanism, Anderson's model for isotype heterojunctions predicts that the reverse current obeys the relation [16]:

$$
I_{R}=B T^{1 / 2} \exp (-E / k T) \exp \left(q V_{1} / k T\right)
$$

where $V_{1}$ is applied reverse voltage drop across a-SiC, $B$ is a constant (independent of temperature and applied reverse voltage), and $E$ is the value of the barrier height that electrons in extended states of the conduction band of a-SiC have to surpass in order to reach the conduction band of $\mathrm{c}-\mathrm{Si}(n)$. However, in the case of a heterojunction like a-SiC/c-Si $(n)$, where one side consists of an amorphous semiconductor, the quantity $E$ should, also, include the a-SiC conductivity activation energy, $E_{\mathrm{a}}$, which is the energy needed for electrons to make a transition from localized gap states near the conduction band to extended states in the band. $V_{1}$ is defined by the relation:

$$
V_{1}=(1-1 / n) V
$$

where $n$ is

$$
n=V / V_{2}
$$

and $V_{2}$ is the applied reverse voltage drop across the c-Si $(n)$. Taking into account that the a-SiC/c-Si $(n)$ isotype heterojunction is a one sided junction [13], where the depletion layer extends, mainly, within the $\mathrm{c}-\mathrm{Si}(n)$, we deduce that $V_{1}$ should be negligible compared with the total applied reverse bias voltage $V$. As a result, the value of the barrier height, $\Delta E_{\mathrm{c}}-V_{b i 1}-V_{1}$, should remain, almost, constant $(\cong 0.18 \mathrm{eV})$, 
independent of the reverse bias voltage and, therefore, $I_{R}$ should also be independent of the reverse bias voltage. On the other hand, $I_{R}$ should be strongly dependent on $T$, because the electron concentration in the extended states of the conduction band of a-SiC, $n$, increases with temperature, according to the relation [10]:

$$
n \propto \exp \left(-E_{\mathrm{a}} / T\right)
$$

Figure 3 shows typical characteristics of $\log \left(I_{R} T^{-1 / 2}\right)$ versus $10^{3} / T$ for two different reverse bias voltages $(V=1 V$ and $4 V)$. It is clear from this figure that a linear relationship is obtained between $\log \left(I_{R} T^{-1 / 2}\right)$ and $1 / T$ for both values of the reverse voltage, which is consistent with eq. (1). It is also clear from this figure that both characteristics have the same slope $(E-(1-1 / n) q V) / k$, where it was found that $(E-(1-1 /$

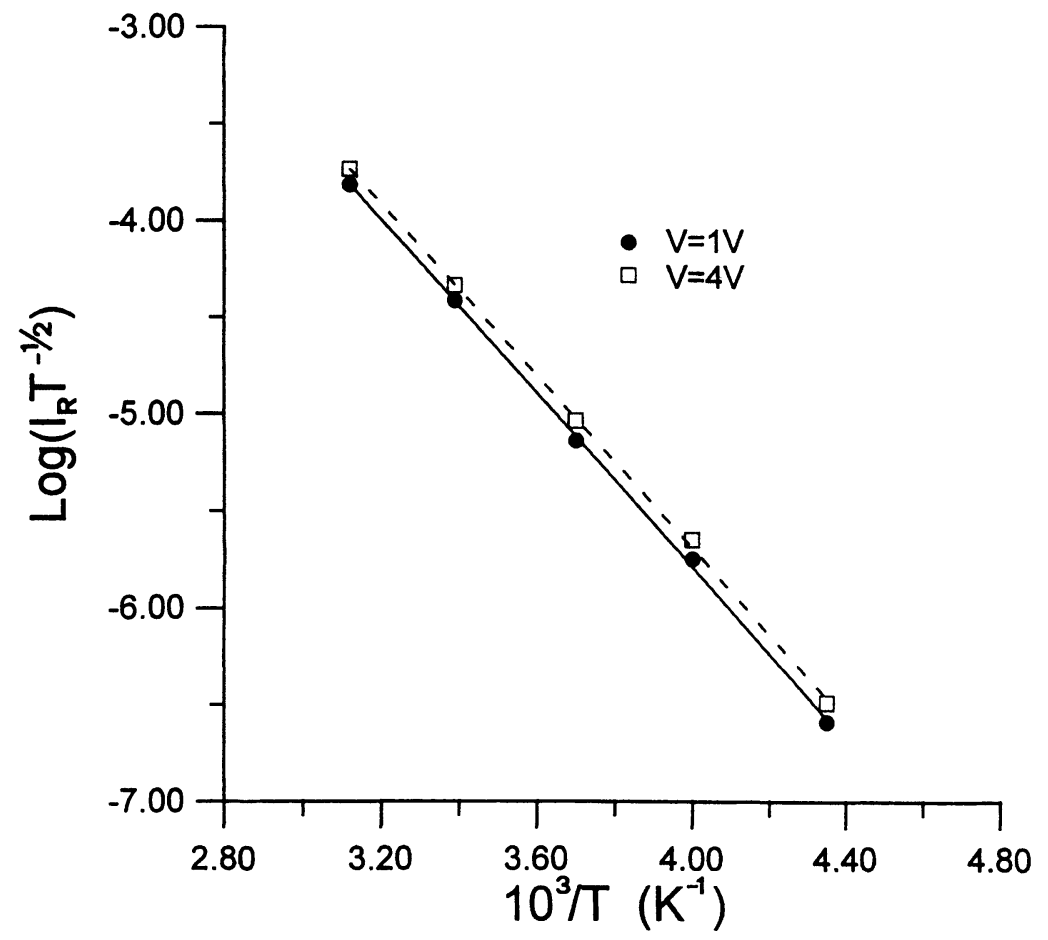

FIGURE $3 \log \left(I_{R} T^{1 / 2}\right)$ of a-SiC/c-Si $(n)$ heterojunctions as a function of $10^{3} / T$ for two different reverse bias voltages (device area $=0.785 \mathrm{~mm}^{2}$ ). 
n) $q V) \cong 0.36 \mathrm{eV}$ ). This suggests that compared with $E$, the quantity $(1-1 / n) q V$ can be ignored, in this range of reverse bias voltages. The observed value of $E(\cong 0.36 \mathrm{eV})$ is exactly equal to the sum of the a-SiC conductivity activation energy $(=0.18 \mathrm{eV})[17]$ and the heterojunction barrier height $\Delta E_{\mathrm{c}}-V_{b i 1}-V_{1}(\cong 0.18 e V)$, suggesting that our previous assumption for $E$ is correct.

The above behavior of a-SiC/c-Si $(n)$ reverse current $I_{R}$ as a function of reverse bias voltage, $V$, and temperature, $T$, leads to high values for the ratio $\Delta V / \Delta T$, which is a measure of the temperature sensitivity of a semiconductor sensor [18]. In order to evaluate the sensitivity of a-SiC/c-Si(n) isotype heterojunctions as a temperature sensor, $V-T$ measurements were carried out using a current source. Figure 4 shows typical $V-T$ characteristics at different values of the reverse current, ranging from $25 \mu \mathrm{A}$ up to $2 \mathrm{~mA}$. It is clear from this figure that the voltage drop across the junction decreases drastically in all $V$ - $T$ characteristics (with the same slope) as the temperature

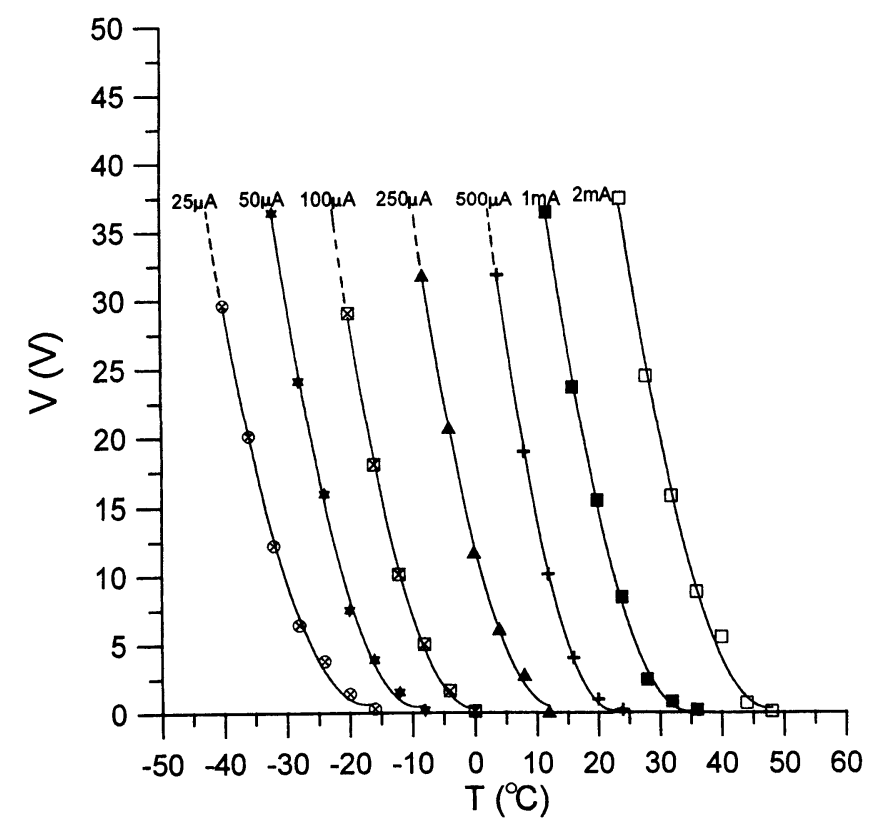

FIGURE 4 Typical reverse bias voltage-temperature $(V-T)$ characteristics of a-SiC/c$\mathrm{Si}(n)$ heterojunctions at different currents (device area $=0.785 \mathrm{~mm}^{2}$ ). 
increases. From these characteristics, the ratio $(\Delta V / \Delta T)$ was calculated and it was found to be $(\Delta V / \Delta T)_{\max } \cong-2.5 V / K$. These values of the temperature sensitivity $(\Delta V / \Delta T)_{\max }$ are much higher than those obtained with bulk semiconductor temperature sensors, where this ratio is about $-1.2 \mathrm{~V} / \mathrm{K}[1,18]$. The above result combined with the fact that the high sensitivity temperature-range of a-SiC/c$\mathrm{Si}(n)$ heterojunctions can be controlled by the reverse current through the device, within the region of values from $230 \mathrm{~K}$ up to $320 \mathrm{~K}$, makes these devices very attractive as temperature sensors.

Differentiating eq. (1) and taking into account eq. (2), the ratio $\partial V / \partial T$ was calculated and it was found to be:

$$
\frac{\partial V}{\partial T}=\frac{\partial V}{\partial I} \frac{\partial I}{\partial T}=\frac{\left(E-V_{1}\right) 1}{(1-1 / n) T}+\frac{k}{(1-1 / n)}
$$

It is clear from eq. (5) that, the sensitivity $\partial V / \partial T$, for a given reverse current, decreases as the temperature, $T$, increases, which is in agreement with experimental results of Figure 4.

\section{CONCLUSIONS}

In the present work the a-SiC/c-Si(n) isotype heterojunction has, for the first time, been studied as a temperature sensor by investigating its electrical properties, under reverse bias conditions, and the main results are as follows:

i) The reverse current-voltage $\left(I_{R^{-}} V\right)$ characteristics at different temperatures (from $230 \mathrm{~K}$ up to $320 \mathrm{~K}$ ) exhibit a saturation behavior, i.e. the $I_{R}$ for a given temperature is almost independent of $V$. On the other hand, the current $I_{R}$ increases drastically with the increase of $T$. The above behavior of $I_{R}$ can be attributed to the form of the energy band levels of the a-SiC/c-Si $(n)$ heterojunction, under reverse bias conditions, as well as to the temperature dependence of the electron concentration in the extended states of a-SiC.

ii) The reverse bias voltage-temperature $\left(V-T^{\prime}\right)$ characteristics at different reverse currents show that $V$ decreases drastically with the increase of $T$, leading to a high value of the temperature 
sensitivity $\left((\Delta V / \Delta T)_{\max } \cong-2.5 V / K\right)$ for all characteristics, so that the high sensitivity temperature-range can be controlled electrically, within the region of values from $230 \mathrm{~K}$ up to $320 \mathrm{~K}$. These observed values of the temperature sensitivity $(\Delta V / \Delta T)_{\max }$ in the moderate temperature are much higher than those for bulk semiconductor temperature sensors. Take, also, into account that a-SiC films can be used as an add-on to the existing Si technology, emerge new possibilities to the fabrication of high sensitivity microsensors.

\section{References}

[1] Schaffer, H. (1984). "Ein empfindlicher silizium-temperatur sensor", $A E U, 38,69$.

[2] Cambell, R. B., Silicon Carbide 1973, "Silicon carbide junction thermistor", Proc. 3rd Int. Conf. On Silicon Carbide, Univ. South Carolina Press, Columbia SC, 1974, p. 611.

[3] Meijer, G. C. (1986). "Thermal sensors based on transistors", Sensors and Actuators A, 10(1-2), 103.

[4] Susuki, M., Murase, K., Kato, N., Tagashi, M. and Hirayama, M. (1986). "Advances of metallic-amorphous silicon-gate FETs in GaAs LSI applications", IEEE Trans. Electron Devices, 33, 912.

[5] Sakaue, M., Murase, M. and Anemiya, Y. (1986). "Self-aligned half-micrometer silicon MASFETs with metallic amorphous silicon gate", IEEE Trans. Electron Devices, 33, 997

[6] Hatalis, M. K. and Greve, D. W. (1987). "High-performance thin film transistors in low-temperature crystallized LPCVD amorphous silicon films", IEEE Electron Device Lett., 3, 361

[7] Dimitriadis, E. I., Georgoulas, N. and Thanailakis, A. (1992). "New a-SiC optically controlled thyristor-like switch", Electronics Lett., 28(17), 1622.

[8] Chang, K. C., Chang, C. Y., Fang, Y. K. and Jwo, S. C. (1987). "The amorphous $\mathrm{Si} / \mathrm{SiC}$ heterojunction color sensitive phototransistor", IEEE Electron Device Lett., $8(2), 64$.

[9] N. F. Mott and E. A. Davis (eds.), "Electronic Processes in Non-crystalline Materials", Clarendon Press, Oxford, 2nd ed., 1979, Ch. 8, p. 273.

[10] M. H. Brodsky (ed.) (1985). “Amorphous Semiconductors", Spinger-Verlag, Berlin Heidelberg, 2nd ed., Ch. 2, p. 120.

[11] Bullot, J. and Schmid, M. P. (1987). "Review on a-SiC", Phys. Stat. Solidi(b), 143, 345.

[12] Magafas, L., Georgoulas, N. and Thanailakis, A. (1992). "Electrical Properties of a $\mathrm{SiC} / \mathrm{c}-\mathrm{Si}(p)$ heterojunctions", Semic. Science and Tech., 7, 1363.

[13] Georgoulas, N., Magafas, L. and Thanailakis, A. (1993). "A study of a-SiC/c-Si(n) isotype heterojunctions", Active and Passive Elec. Comp., 16, 55.

[14] Anderson, R. (1962). "Experiments on Ge-GaAs heterojunctions", Solid-State Electron, 45(9), 341.

[15] J. D. Joannopoulos and Lucovsky (eds.) (1984). "The physics of Hydrogenated amorphous Silicon II", Springer-Verlag, Berlin Heiberg, Ch. 4, p. 170.

[16] Sharma, B. L. and Purohit, R. K. (1974). "Semiconductor Heterojunctions", Int. Series of Monographs in the Science of Solid State, 5, Pergamon, New York, Ch. 2, p. 25 . 
[17] Magafas, L., Georgoulas, N., Girginoudi, D. and Thanailakis, A. (1991). "The dependence of electrical and optical properties of r.f. sputtered amorphous siliconcarbon alloy thin films on substrate temperature and hydrogen flow rate", Phys. State. Sol.(a), 124, 143.

[18] Schaffer, H. (1986). Temperaturverhalten von barrierengesteuerten Silizium-pnpdioden, $\mathrm{Ph}$. D. Thesis Technichal University of Munich. 

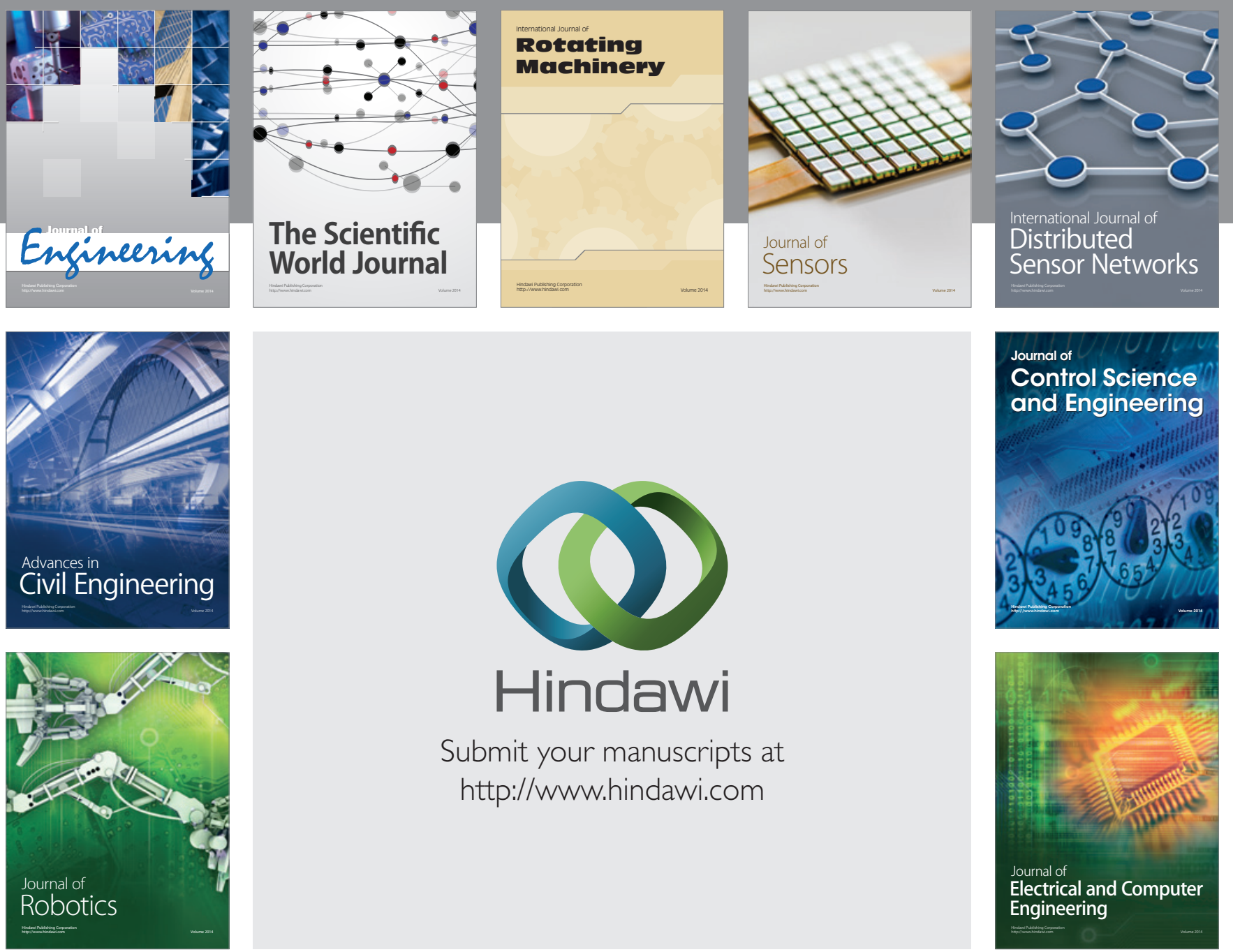

Submit your manuscripts at

http://www.hindawi.com
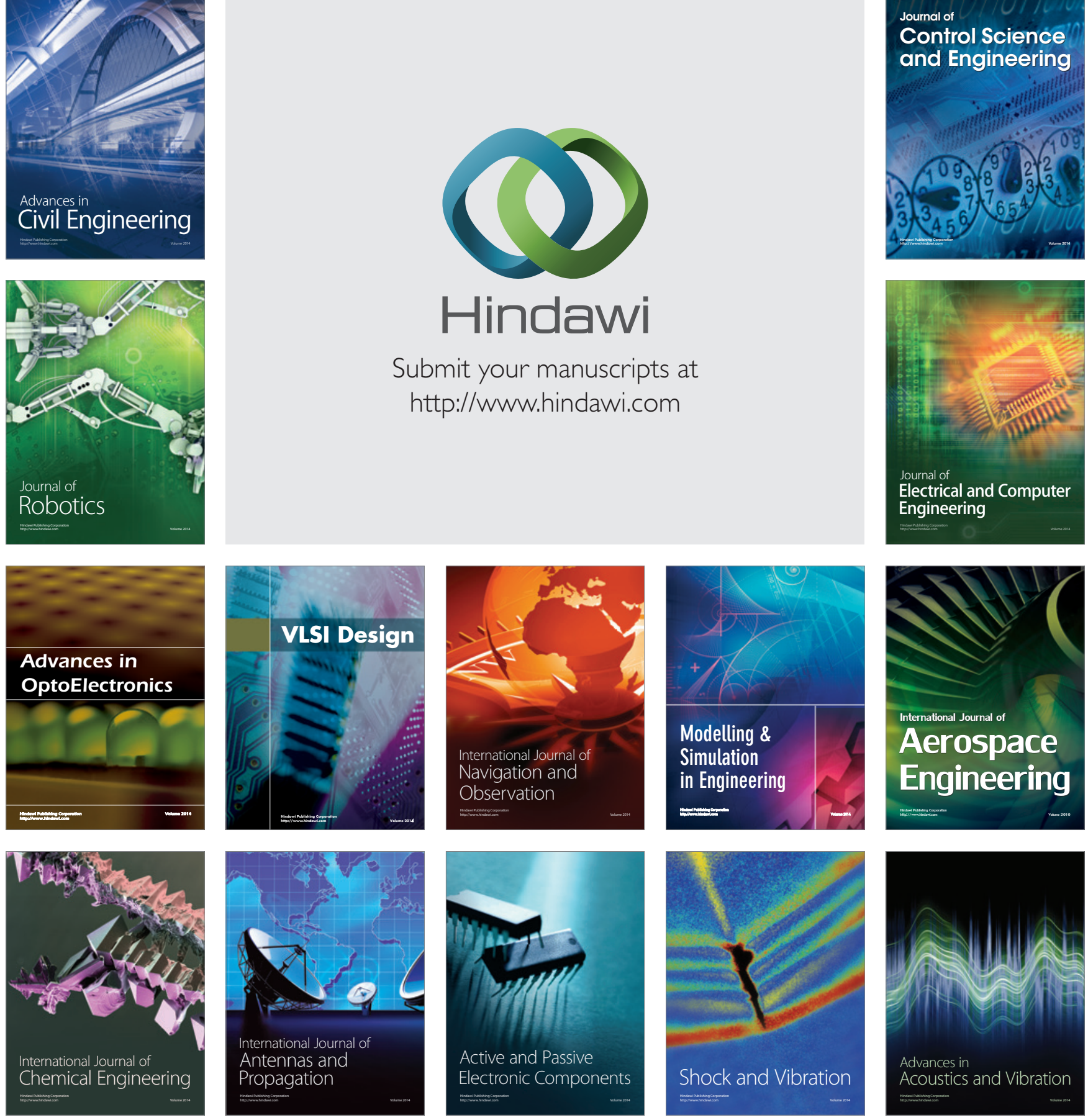